\title{
The Effect of Cosmic Rays on Cometary Nuclei. I. Dose Deposition
}

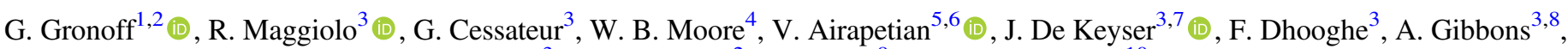 \\ H. Gunell ${ }^{3}$, C. J. Mertens ${ }^{2}$, M. Rubin ${ }^{9}$ (D), and S. Hosseini ${ }^{10}$ \\ ${ }^{1}$ Science Systems and Application Inc., Hampton, VA, USA; Guillaume.P.Gronoff@nasa.gov \\ ${ }^{2}$ NASA Langley Research Center, Hampton, VA, USA \\ ${ }^{3}$ Royal Belgian Institute for Space Aeronomy (BIRA-IASB), Brussels, Belgium \\ ${ }^{4}$ Hampton University, Hampton, VA, USA \\ ${ }^{5}$ American University, Washington, DC, USA \\ ${ }^{6}$ NASA GSFC/SEEC, Greenbelt, MD, USA \\ ${ }^{7}$ Centre for Mathematical Plasma Astrophysics, Heverlee, Belgium \\ ${ }^{8}$ Université Libre de Belgique, Brussels, Belgium \\ ${ }^{9}$ Physikalisches Institut, University of Bern, Bern, Switzerland \\ ${ }^{10}$ Jet Propulsion Laboratory, Pasadena, CA, USA \\ Received 2019 November 5; revised 2019 December 20; accepted 2019 December 28; published 2020 February 14
}

\begin{abstract}
Comets are small bodies thought to contain the most pristine material in the solar system. However, since their formation $\approx 4.5 \mathrm{~Gy}$ ago, they have been altered by different processes. While not exposed to much electromagnetic radiation, they experience intense particle radiation. Galactic cosmic rays and solar energetic particles have a broad spectrum of energies and interact with the cometary surface and subsurface; they are the main source of space weathering for a comet in the Kuiper Belt or in the Oort Cloud, and also affect the ice prior to the comet agglomeration. While low-energy particles interact only with the cometary surface, the most energetic ones deposit a significant amount of energy down to tens of meters. This interaction can modify the isotopic ratios in cometary ices and create secondary compounds through radiolysis, such as $\mathrm{O}_{2}$ and $\mathrm{H}_{2} \mathrm{O}_{2}$ (Paper II). In this paper, we model the energy deposition of energetic particles as a function of depth using a Geant 4 application modified to account for the isotope creation process. We quantify the energy deposited in cometary nucleus by galactic cosmic rays and solar energetic particles. The consequences of the energy deposition on the isotopic and chemical composition of cometary ices and their implication on the interpretation of cometary observations, notably of $67 \mathrm{P} / \mathrm{Churyumov}$ Gerasimenko by the ESA Rosetta spacecraft, will be discussed in Paper II.
\end{abstract}

Unified Astronomy Thesaurus concepts: Comets (280); Galactic cosmic rays (567); Cosmic rays (329); Solar energetic particles (1491); Pre-biotic astrochemistry (2079)

\section{Introduction}

Comets formed in the early stage of the solar system and, today, reside in two main reservoirs, the Kuiper Belt (KB) scattered disk (355 au) and the Oort Cloud (OC; 2000-200,000 au). Orbital perturbations (for instance, a collision or the gravitational force exerted by Jupiter) bring some comets closer to the Sun. If close enough to the Sun, their nuclei are heated by sunlight and cometary ice sublimates into space, while refractory grains are set free. It is during this stage that spacecraft can sample in situ cometary material as the ESA/Rosetta probe did from 2014 August to 2016 September around comet 67P Churyumov/Gerasimenko (referred to as 67P in the following). 67P is a $\mathrm{KB}$ comet that has been gravitationally displaced into the inner solar system by a close encounter with Jupiter in 1959 (Maquet 2015). It is now orbiting with a semimajor axis of 3.46 au (Carusi et al. 1985). The comet is estimated to lose from a few up to tens of meters of surface material during each orbit (Bertaux 2015; Keller et al. 2015). It should thus have lost several tens of meters on average since insertion in its current orbit, with locally higher erosion rates, depending on the position on the surface of the nucleus (Groussin et al. 2015). The loss may actually be higher since the comet was already outgassing when it reached the Jupiter region during its pre-1959 orbit (Matonti et al. 2019).

Consequently, the neutral gas measured in situ in the coma of comet 67P by the ROSINA experiment (and also MIRO, VIRITS, and Alice) on board Rosetta likely originated several tens of meters beneath the primordial surface of the comet. ROSINA observations provided evidence that this comet is formed from pristine material that has not been significantly altered after its formation in the first $\mathrm{Myr}$ of the solar nebula stage. The high abundance of super-volatiles like $\mathrm{CO}$ and $\mathrm{CO}_{2}$ (Le Roy et al. 2015), the detection of argon (Balsiger et al. 2015), of molecular nitrogen (Rubin et al. 2015), of molecular oxygen (Bieler et al. 2015), of a high $\mathrm{D} / \mathrm{H}$ in $\mathrm{HDO} / \mathrm{H}_{2} \mathrm{O}$ and $\mathrm{D}_{2} \mathrm{O} / \mathrm{HDO}$ and $\mathrm{HDS} / \mathrm{H}_{2} \mathrm{~S}$ (Altwegg et al. 2015, 2017), and of hydrogen halides (De Keyser et al. 2017; Dhooghe et al. 2017), coupled with the low density, high porosity, and homogeneity of the nucleus (Pätzold et al. 2016) and the absence of signatures of aqueous alteration (see Capaccioni et al. 2015; Davidsson et al. 2016; Quirico et al. 2016; Bardyn et al. 2017) all indicate that comet 67P formed at low temperature and did not experience any substantial global-scale heating after its formation. This suggests that $67 \mathrm{P}$ is representative of the solar nebula material from which the solar system had formed. This has strong implications not only for how the measurements made in cometary environments can be used to constrain the protosolar environment but also for the contribution of comets to Earth's composition. For instance, the measurement of the $\mathrm{D} / \mathrm{H}$ isotopic ratio in 67P (Altwegg et al. 2015) suggests that comets cannot be considered as the main source of water on Earth. The discovery of significant amounts of $\mathrm{O}_{2}$ in comets (Bieler et al. 2015) was not predicted by astrochemical models and challenges our understanding of the chemistry of molecular 
clouds and of the protosolar nebula. However, the Jupiterfamily comets (JFCs; which include 67P) are a diverse groups. Indeed, even if Giotto measurements indicate that comet $1 \mathrm{P} /$ Halley contains similar amounts of $\mathrm{O}_{2}$ (Rubin et al. 2015), different $\mathrm{D} / \mathrm{H}$ ratios (lower than observed for $67 \mathrm{P}$ and compatible with the $\mathrm{D} / \mathrm{H}$ ratio in the Earth's oceans) have been measured for other JFC comets like Hartley 2 (Balsiger et al. 2015) and 46P/Wirtanen (Lis et al. 2019). The causes for this diversity may be already present at the formation of these comets or may result from a different evolution after their formation.

Indeed, there is evidence that some processes alter cometary material after their formation (e.g., Stern 2003; GuilbertLepoutre et al. 2015 and references therein) without inducing a significant large-scale heating of the nucleus. The main reservoirs of comets, the $\mathrm{KB}$ and $\mathrm{OC}$, are sufficiently far from the Sun so that comets receive little heat. In addition, their small size (a few tens of kilometers or less) prevents comets from transforming by internal (radiogenic) heating (Mousis et al. 2017). However, the thermal environment of comets may have varied under the effect of passing stars and of supernova explosions in the vicinity of the solar system. Stern \& Shull (1988) consider it very likely that OC comets have been heated at least once by a luminous $O$ star passing in the vicinity $(<5 \mathrm{pc})$ of the solar system. This can raise the temperature of the OC from $5-6 \mathrm{~K}$ to $\sim 16 \mathrm{~K}$ during a period of the order of $\sim 3 \times 10^{4} \mathrm{yr}$. For $\mathrm{KB}$ comets the estimated temperature increase is lower $(\sim 1 \mathrm{~K})$ as their equilibrium temperature is higher $(30-60 \mathrm{~K})$ due to their location at a shorter heliocentric distance. OC comets may also have experienced several, shortterm $(\sim 0.1 \mathrm{yr})$ temperature increases of a few tens of Kelvin due to nearby supernovae (Stern \& Shull 1988). Due to the short duration of supernovae explosions, this type of heating would mostly affect the first meter below the surface of a cometary nucleus while the heat from a passing $\mathrm{O}$ star can penetrate down to a few tens of meters owing to its longer duration. Heating by passing stars and supernovae in the OC regions may have removed some part of the most volatile elements from their surface/subsurface.

While OC comets may have experienced collisions during their ejection from the inner young solar system to the OC (Stern \& Weissman 2001), the OC is essentially a collisionless environment (Stern 1988) due to its low density and to the low orbital velocity in this region (see Stern 2003 and references therein). It is thus likely that OC comets have not been significantly altered by collisions. KB comets may have experienced more frequent collisions after they formed (e.g., Duncan et al. 2004) as both the density and orbital velocities are higher in this region, which should result in a higher collision rate than in the OC. The collisional history of $\mathrm{KB}$ comets is still debated. Several scenarios have been proposed to explain the formation of bilobate-shaped comets like 67P. Davidsson et al. (2016) argue that Rosetta observations of comet 67P are in better agreement with a comet formation by agglomeration of primordial rubble-piles that remained in the protosolar nebulae after trans-Neptunian object formation. This process should be slow enough, between $\sim 2.2$ and $7.7 \mathrm{Myr}$ after the incorporation of $\mathrm{Ca}-\mathrm{AL}-$ rich inclusions from the protosolar nebula in $67 \mathrm{P}$, in order to avoid thermal heating by short-lived radionuclides (Mousis et al. 2017). Observations by the OSIRIS camera on board Rosetta evidenced that $67 \mathrm{P}$ consists of two different objects that have formed a contact binary (Massironi et al. 2015). Bilobate comets are relatively common, and Davidsson et al. (2016) suggested that the final stage of comet formation results in merging between lobes and that comets are unlikely to experience destructive collisions. Other authors come to the opposite conclusion: Morbidelli \& Rickman (2015) and Rickman et al. (2015) estimated the probability that $67 \mathrm{P}$ survived collisions to $10^{-4}$ if formed early in the protosolar nebula. Jutzi \& Benz (2017) suggest that bilobate comets like 67P may have formed due to subcatastrophic collisions occurring later, which would not lead to an alteration of the nucleus in agreement with 67P properties. According to Jutzi et al. (2017) comets may have experienced several shape-changing collisions and the current shape of comet 67P would thus result from the last major shape-forming impact that most probably occurred within the last Gyr. Finally, Schwartz et al. (2018) suggest that catastrophic collisions may have occurred and that the geological features of comets are not primordial.

Comets also interact with the interstellar medium (ISM) after their formation via two competing processes: accretion of ISM material and erosion caused by the impact of high-velocity ISM grains. According to simulations, the latter could be dominant for OC comets and could lead to a significant erosion of the cometary surface in the range of several meters (Mumma et al. 1993). However, erosion by ISM grains is a complex process depending for instance on the ISM grain composition and three-dimensional structure, and such erosion estimates are thus highly speculative (Belyaev \& Rafikov 2010).

One of the major candidates for altering cometary material are cosmic rays. Indeed, comets are constantly bombarded by galactic cosmic rays (GCRs). The bulk of the cosmic rays, including protons below $1 \mathrm{GeV}$, are able to penetrate into the surface layers of the comet (Johnson 1991). However, higherenergy cosmic rays penetrate deeper. In addition, comets are bombarded by stellar particles at lower energies such as gamma-ray bursts (Johnson 1991).

The major goal of this paper is to quantify the energy deposited by cosmic rays and solar energetic particles (SEPs) in comets. In Section 2, we describe the CometCosmic model, based on Geant4, that is at the core of this study. We also describe the modeled composition of the comet and the the GCR spectra used. In Section 3, the energy deposition in the comet by each source of particles is discussed for both $\mathrm{KB}$ and OC comets. We discuss the effect of the early solar system irradiation on the energy deposited in comets and the effect of the high- $Z$ cosmic rays in Section 4. In a companion paper (Maggiolo et al. 2020, hereafter Paper II), we will discuss the effects of energy deposition by GCRs on the composition and structure of cometary nuclei, and how this can change our interpretation of space mission data.

\section{Model and Simulations}

The simulation of the cosmic-ray impact on comets was performed using CometCosmic, a new model based on the GEometry ANd Tracking (Geant4; Agostinelli et al. 2003) library. For the present calculations, we used version 4.9.6. This library allows us to compute the transport of energetic particles -including the electromagnetic cascade, neutron creation and capture, and isotopic changes-in a given medium. Based on the recommendation for the library usage and on our previous experience with Geant4 simulation (Gronoff et al. 2009; Sheel et al. 2012; Gronoff et al. 2015, 2016), we used the physics list 
QGSP_BIC_HP because it is the best suited to analyze the fragmentation of atomic nuclei and therefore to study the formation of isotopes. In addition, we modified the neutron model to be able to count the atomic nuclei created, allowing to fully evaluating the variation of the isotopic ratio due to cosmic rays (Pavlov et al. 2014). We simulated a $5 \mathrm{~km}$ radius spherical comet, with density 0.5378 following the estimate for $67 \mathrm{P}$ (Pätzold et al. 2019), composed of water ice (of isotopic composition ${ }^{16} \mathrm{O}$ and ${ }^{1} \mathrm{H}$ ) and $\mathrm{SiO}_{2}$ with a dust (refractory material)/water ratio of 4 in mass (i.e., $5 \mathrm{H}_{2} \mathrm{O}$ for $6 \mathrm{SiO}_{2}$; Rotundi et al. 2015). This ratio has been further studied and is still debated; it has been estimated from higher than six (Fulle et al. 2016) to lower than one (Läuter et al. 2018). For the study of the ${ }^{15} \mathrm{~N}$ variation (Paper II), we assume an initial $\mathrm{N}$ mixing ratio of $10^{-3}$ (Iro et al. 2003); $\mathrm{N}$ is not included in the cosmicray simulation to reduce the required amount of processor time. No complex surface material is assumed in this model; the refractory material (dust) is taken to be exclusively $\mathrm{SiO}_{2}$, and the comet is considered as homogeneous, i.e., its porosity is homogeneous. (In actual comets, the heterogeneity leads to locations being more or less eroded, and leads to locations more pristine than others, which increases the chance of seeing GCRweathering effects in observations.)

For our simulations, the GCRs have been separated into different groups with a dedicated mass and charge to accelerate the computation (Velinov \& Mateev 2008): proton, alpha, M group $(Z=7, A=14$ in our simulations, for a group that contains mainly carbon and oxygen nuclei), $\mathrm{H}$ group $(Z=12$, $A=24$, mainly silicon nuclei), and $\mathrm{VH}(Z=26, A=56$, mainly iron nuclei). Please note that the energy is in $\mathrm{GeV}$, and not in $\mathrm{GeV} /$ nucleon, to highlight the importance of the mass, and to be consistent with the other figures. We performed the Geant 4 simulations for each of these groups between $1 \mathrm{keV}$ and $1 \mathrm{TeV}$, using two grids with exponentially distributed energies. The lower energies extend, i.e., $1 \mathrm{keV}-1 \mathrm{GeV}$ allowed us to show the influence of the solar wind-and interstellar particles - on the first few centimeters of the comet, as well as the effects of the SEPs. For that, 100 energies were selected on an exponentially increasing grid, and the products were computed on a $1 \mathrm{~cm}$ depth resolution grid. The higher energies, $1 \mathrm{GeV}-$ $1 \mathrm{TeV}$, were optimized to the computation of the actual GCR and SEP influence; 50 energy bins were computed with a $1 \mathrm{~m}$ depth resolution comet. The $1 \mathrm{TeV}$ upper limit was determined empirically: above that energy, the increase in dose deposition is negligible in the comet, and while it can impact the lower depth (below $80 \mathrm{~m}$ ) its overall effect on the comet lifetime (see Paper II) is negligible. For all these energies and particle groups, eight angles were taken to account for the spherical geometry of the comet. The number of particle shots in the Monte Carlo simulation was optimized to have less than a $5 \%$ uncertainty on the deposited dose at the peak, computed statistically. The error bars presented in the different figures have been computed using statistical analysis and appropriately weighted (Gronoff 2009; Gronoff et al. 2009).

Figure 1 shows the results of the simulation of energy deposition per unit volume in the comet due to a proton flux (or fluence) irradiation of a given energy as a function of the depth inside the comet. This is an average profile since the depth depends upon the impact angle; our simulations involve integrating the isotropic flux over eight angles for better depth accuracy, which is crucial in the spherical geometry (Gronoff et al. 2011; Norman et al. 2014; Gronoff et al. 2015).

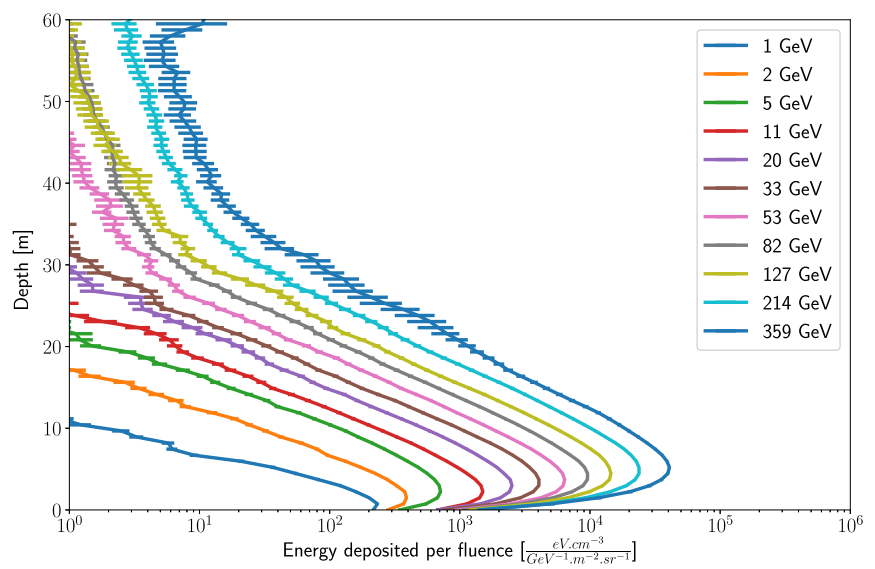

Figure 1. Deposition of energy by protons of different initial energy as a function of the depth and incident fluence in the comet nucleus. The more energetic the proton, the deeper the peak and the higher its intensity. Only a few initial proton energies are shown here, but the whole simulation considers a larger set of energies and more nuclei. The deposition is in dose per fluence, i.e., we have a dose in $\mathrm{eV} \mathrm{cm}^{-3}$ if the fluence of a given particle, in $\mathrm{GeV}^{-1} \mathrm{~m}^{-2} \mathrm{sr}^{-1}$, is 1 ; this is equivalent to a dose per second per unit flux.

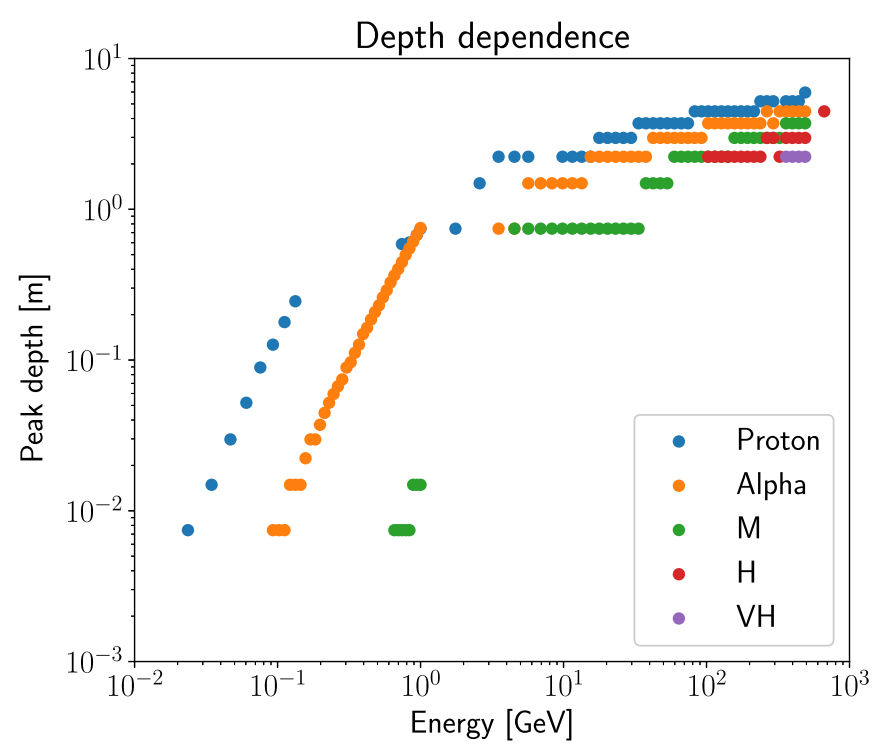

Figure 2. Peak energy deposition depth as a function of the energy of the primary particle computed by CometCosmic. Discontinuities are due to the resolution of the model, set at $1 \mathrm{~cm}$ depth below $1 \mathrm{GeV}$ and $1 \mathrm{~m}$ above.

The figure shows that energy deposition per unit volume peaks at a depth of $5 \mathrm{~m}$ for $300 \mathrm{GeV}$ protons. Figure 2 shows the dependence of the peak production depth as a function of energy and precipitating particle type. This shows that, while particles with energy lower than $1 \mathrm{GeV}$ have a peak energy deposition depth below $1 \mathrm{~m}$, particles with higher energies penetrate deeper into the comet and deposit their energy in the first few tens of meters inside the nucleus. From 1 to $100 \mathrm{GeV}$, while the incident energy varies by two orders of magnitude the peak energy deposition varies only a little (a few meters).

We used two cosmic-ray flux models in our study, as shown in Figure 3. The first one, the most realistic for 67P, considers the flux of cosmic rays in the KB region. The second one takes into account the local interstellar spectra (LIS) to simulate the GCR flux in the OC region. 

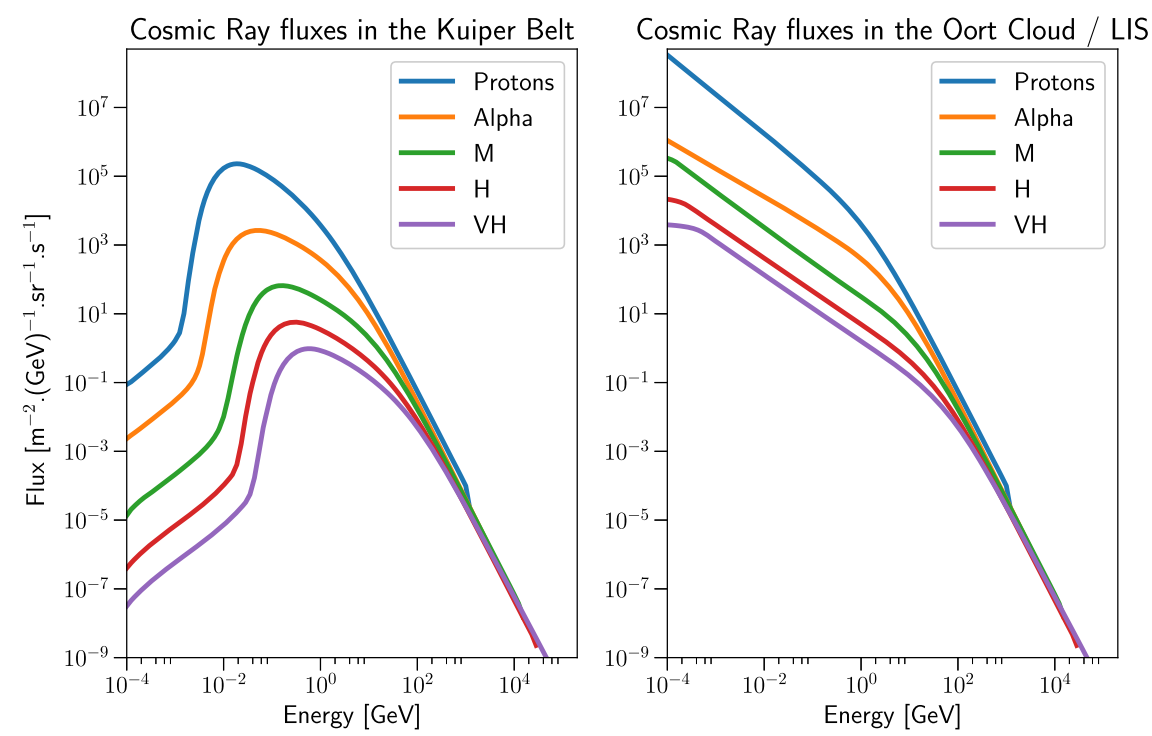

Figure 3. Galactic cosmic-ray flux in the Kuiper Belt and in the Oort Cloud (local interstellar spectrum) as modeled by the Badhwar-O'Neill model. The solar modulation in the Kuiper Belt leads, as expected, to a large decrease of the flux at low energy.

For computing these fluxes, we used the modified Badhwar \& O'Neill (1992) model (hereafter B-O) H-BON10 (Mertens et al. 2013). While other modifications of B-O exist (e.g., Matthiä et al. 2013; O'Neill et al. 2014), this is the only version of the B-O model that allows the radial distance to be specified as an input parameter. It also allows the solar modulation parameter to be parameterized by real-time, high-latitude neutron monitor data. The solar modulation parameter for the H-BON10 model is shown in Mertens et al. (2013) over a $50 \mathrm{yr}$ period. For the calculations at $40 \mathrm{au}$, we used a solar modulation of $1000 \mathrm{MV}$ so it reflects the average solar modulation. For the LIS, we used a 0 MV modulation; the distance having no impact in that case. The H-BON10 model gives the fluxes for all the ion; the different ions fluxes were added as a function of their charge to give the different group fluxes. M corresponds to ions from $Z=3$ to $Z=10 ; \mathrm{H}$ to from $Z=11$ to $Z=20$; and $\mathrm{VH}$ to $Z>20$. Our comparison for planetary atmospheres between planetocosmics and HZETRN (Gronoff et al. 2011, 2015) shows that this approximation is valid for our kind of study.

The difference in irradiation between a KB comet and an OC comet mainly results from the shielding by the heliospheric magnetic field. As a result, the flux of particles below $\sim 1 \mathrm{GeV}$ is attenuated in the KB region. The main assumption for this model is that the GCR flux has not significantly changed over $4.5 \mathrm{Gyr}$, and that the comet stayed in a region where the solar modulation of the GCR was stable (Poluianov et al. 2018). This very strong hypothesis will be discussed in Section 3.3. The anomalous cosmic-ray flux (Giacalone et al. 2012; Simnett 2017) is also of interest for comet irradiation studies, their fluxes can be found in Cummings \& Stone (2007); however, when compared to the B-O model fluxes, they are of lower intensities and are therefore not included in our calculations. The model does not take into account the irradiation by gamma-ray bursts and solar photons as these particles/events have negligible effects below the first meter inside cometary nuclei according to our preliminary calculation using CometCosmic.

A second source of high-energy particles are the SEP events. While they are rare, their influence over the solar age has varied

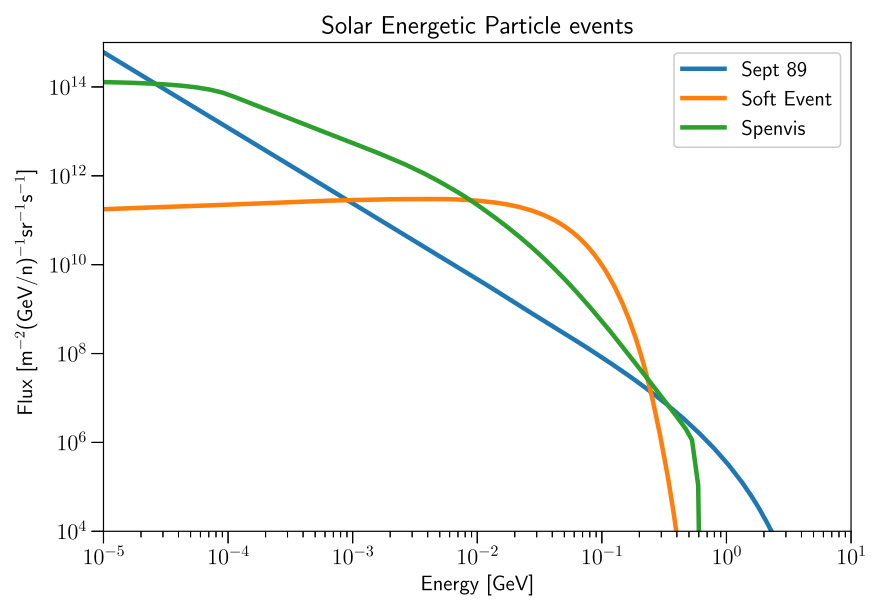

Figure 4. Solar energetic particle event fluxes modeled in our calculations. These fluxes, which correspond to reconstructions of historical events, highlight different intensities and hardness (high-energy tails) encountered in the different spectra. The soft event is based on the work of Smart et al. (2006), who tried to reconstruct a Carrington event. The validity of this spectra as a Carrington reconstruction has later been rejected, as discussed in Wolff et al. (2012) and Kovaltsov et al. (2014), and is used here as an example of an intense spectra with fewer particles above $100 \mathrm{MeV}$. The "SPENVIS" event is the worst event of 1989 October as modeled by the European Space Agency's Space Environment Information System website SPENVIS ${ }^{11}$; the 1989 September event is based on the work of Sheel et al. (2012).

a lot (Airapetian et al. 2016; Fu et al. 2019). In this paper, we study the impact that an SEP would have on a comet. Several SEPs flux models have been implemented in our model, taken from Norman et al. (2014) and Gronoff et al. (2015) and are shown in Figure 4. These SEP event flux profiles were computed for a location at $1 \mathrm{au}$, which is far from the conditions encountered by the comet. If one considers a distance of $50 \mathrm{au}$, the reduction in flux is of the order of 2500 , based on an $R^{-2}$ decrease (which is an approximation since magnetic fields can affect the dispersion with distance; Rodríguez-Gasén et al. 2014). These SEP fluxes are

\footnotetext{
${ }^{11}$ https://www.spenvis.oma.be/
} 


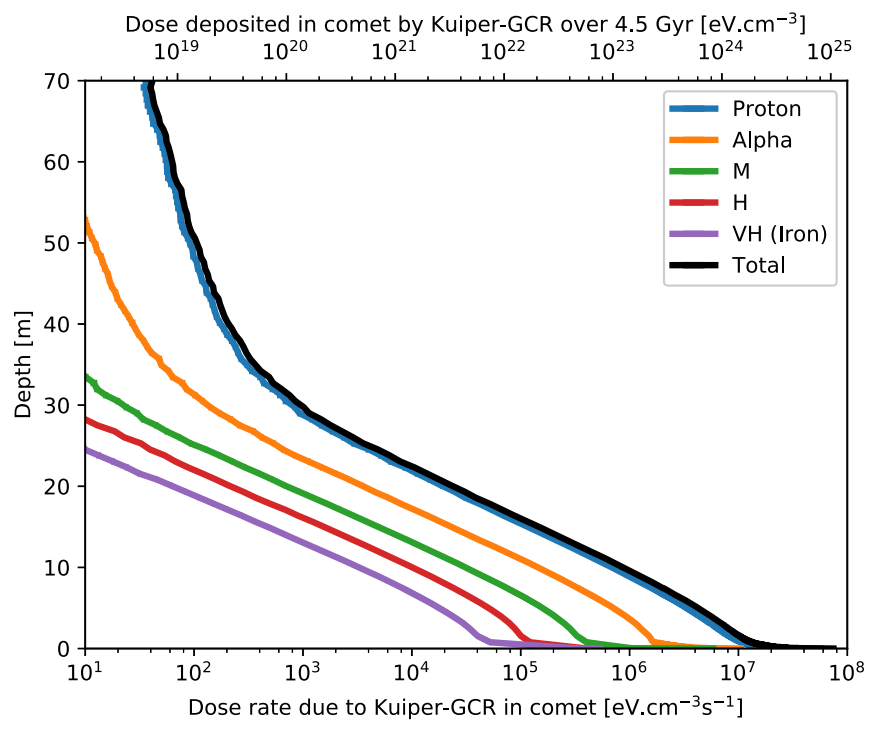

Figure 5. Dose deposited per second in the comet per cubic centimeter for the 67P conditions (in the Kuiper Belt), as a function of the incident GCR nuclei groups.

comparable to those the comet must have suffered during the early phase of the solar system (Airapetian et al. 2016); therefore, we use them for the present calculation.

\section{Energy Deposition in the Comet}

There are many effects of dose deposition. For example, one can expect a $35 \mathrm{eV}$ dose deposition to lead to ionization, provided that the particle depositing energy is at an energy above this threshold, which is the case in the present study. Ionization and dissociation/excitation of molecular species initiate chemical reactions for the creation of molecular species not initially present in the comet. Several works on ice ionization (Johnson 1991; Paper II and references therein) have shown the chemical effects of dose deposition. One can expect a dose rate as small as $100 \mathrm{eV} \mathrm{cm}^{-3} \mathrm{~s}^{-1}$ to create species in nonnegligible quantities if sustained over billions of years as shown in Paper II.

\subsection{Galactic Cosmic Rays}

Figure 5 presents the dose deposited in the comet per cubic centimeter per second for the conditions encountered in KB. Figure 6 represents the dose deposited in the case of an OC comet. In both cases, the energetic protons are responsible for energy deposition up to $10^{8} \mathrm{eV} \mathrm{cm}^{-3} \mathrm{~s}^{-1}$ in the first centimeters. The difference between these two cases lies only in the first meter below the surface where for OC conditions more energy is deposited as, contrary to the $\mathrm{KB}$ region, the shielding of low-energy GCRs by the heliospheric magnetic field is negligible. This effect is demonstrated in Figure 7. In the following, we will therefore only show figures for the $\mathrm{KB}$ conditions. The effect of the refractory material/water ratio has an impact on the manner the energy will be dissipated in the comet: which kind of ionization and which kind of isotopes will be created. This ratio will therefore mainly change the effects modeled in Paper II. The porosity of the comet will change the depth at which the energy deposition will happen: the depth could be changed into a column density as a first approximation to evaluate the effects of porosity, but the small size of the comet implies that spherical effects are quickly important if the porosity is changed by factors greater than $\approx 2$.

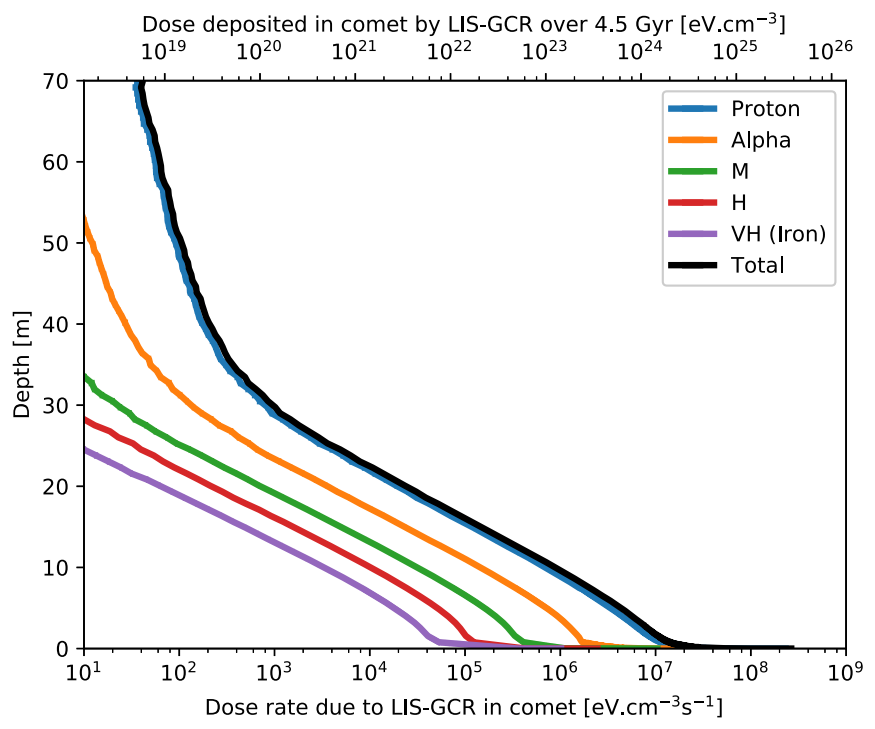

Figure 6. Dose deposited per second in the comet per cubic centimeter for the Oort Cloud (LIS) conditions, as a function of the GCR nuclei group precipitating.

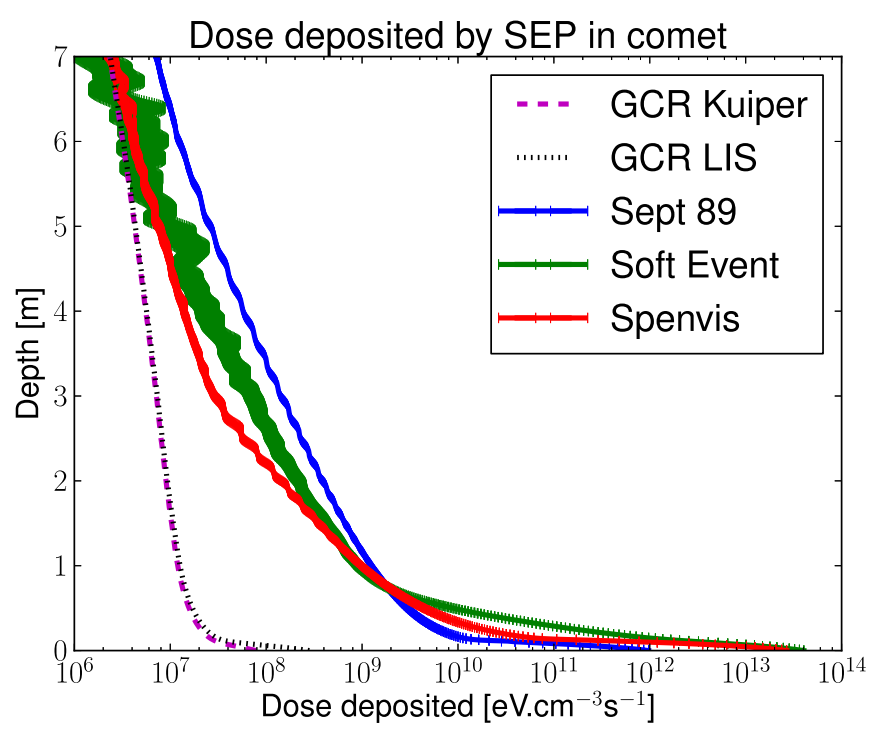

Figure 7. Dose deposited in the comet per cubic centimeter per second for the $67 \mathrm{P}$ conditions, as a function of the SEP event shown in Figure 4. (This corresponds to a Kuiper Belt comet subjected to a super-Carrington event or a comet in the inner solar system subject to current SEP events.) The GCR doses are displayed to show how they become the dominant ionization source, in all conditions, deeper than $\approx 8 \mathrm{~m}$.

The cumulative impact after $4.5 \mathrm{Gyr}$ can be seen at the top of the Figures 5 and 6 (top $x$-axis). The dose computed is integrated over $4.5 \mathrm{Gyr}$ by assuming that the cosmic-ray flux in Figure 1 does not vary in time. Therefore, a deposition dose rate of $10^{8} \mathrm{eV} \mathrm{cm}^{-3} \mathrm{~s}^{-1}$ corresponds to the energy deposition of $\approx 10^{25} \mathrm{eV} \mathrm{cm}^{-3}$ This assumption will be discussed in more depth in Section 3.3. As expected, the protons produce most of the dose, with a deposition of the order of $10^{25} \mathrm{eV} \mathrm{cm}^{-3}$ (i.e., $1.6 \mathrm{MJ} \mathrm{cm}^{-3}$ ) in the first meter below the surface.

\subsection{Effects of High-Z}

The alpha and high- $Z$ cosmic rays (oxygen nuclei, carbon nuclei, and higher-mass nuclei such as $\mathrm{Fe}$ ) are a minority of the cosmic rays; however, their mass compensates their rarity 
(Gronoff et al. 2011), and the dissociation of nuclei can account for the creation of more isotopes (G. Gronoff et al. 2020, in preparation). As seen in Figures 5 and 6, the dose deposition in the comet by the alpha and high- $Z$ particles is small in comparison with proton deposition. These particles are, however, of interest for studying the deposition of heavy ions and the variation of isotopic ratio due to the different nuclear reactions (spallations, neutron capture) over the $4.5 \mathrm{Gyr}$ lifetime of the comet.

\subsection{Additional Radiation Sources in the Early Solar System}

The question of the stability of the GCR spectra in time is a major unknown for studying space weather effects during eons. The amount of cosmic rays hitting the comet varied in time, notably because of the early Sun's activity but also because of supernovae in the vicinity of the solar system. We used the modern spectra as an average for our study for the following reasons:

(1) The early solar system is believed to have been exposed to more charged particle irradiation, as shows by meteoritic ${ }^{26} \mathrm{Al}$ anomalies (Feigelson \& Montmerle 1999; Kööp et al. 2018). The major particle flux possible comes from the superflare associated coronal mass ejections that drive high fluence SEP events (also referred as solar cosmic rays in this context), with fluence on the order of $10^{30}$ protons (Airapetian et al. 2019; Fu et al. 2019). Indeed, simulations shows that nearby supernovae could not explain the production of ${ }^{26} \mathrm{Al}$ observed in the meteorites (Portegies Zwart et al. 2018). The energies of such solar cosmic rays would be below $1 \mathrm{GeV}$ (Airapetian et al. 2016; Fu et al. 2019, and references therein), and therefore would be of negligible influence in the deeper layers of the comet. They affect only on the first meter below the surface.

(2) Considering explosion of different supernovae in the vicinity of the early solar system (Torres et al. 2012), it is difficult to estimate the corresponding total particle fluence. Most of the energy from such an event would be transported by particles below $1 \mathrm{MeV}$, and therefore would impact the first centimeter of the comet. Higherenergy particles would impact the comet like GCRs but for a relatively small period of time. In any case, the first meter of a comet may be the first part of the comet to erode as it enters the inner solar system provided that it has not been eroded by high-velocity ISM grains earlier on. If the first layers were subject to particles from supernovae explosions, the change in isotopic ratio of non-diffusing species would mimic the GCR effects (because of similar spectra in the $1-100 \mathrm{GeV}$ range), while if it were Sun related, the change would be skewed toward the first meter. The main advantage of doing such a study in a comet, with respect to an asteroid, is that a pristine comet would have been less affected by later solar events, and would have suffered fewer collisions.

\subsection{Solar Wind and Low-energy Interstellar Charged Particles}

The solar wind is a continuous flow of ions and electrons, mostly containing $\mathrm{H}^{+}$ions (96\% on average), alpha particles (4\% on average), and a minor fraction of highly charged heavy ions (Wurz 2005). Typical solar wind ion energy is of the order of $1 \mathrm{keV}$, and thus they deposit most of their energy in the first centimeter below the nucleus surface (see Figure 2).

The average properties of the solar wind at heliocentric distance between 25 and 39 au have been estimated by Bagenal et al. (2015) using data from Voyager 2 (the measurements were made between 1988 and 1992). They obtain a median value of $0.0058 \mathrm{~cm}^{-3}$ for the density and of $429 \mathrm{~km} \mathrm{~s}^{-1}$ for the velocity. Considering that these values are representative of solar wind conditions in the $\mathrm{KB}$, we can estimate the solar wind flux reaching $\mathrm{KB}$ comets to be of the order of $2.4 \times$ $10^{7} \mathrm{eV} \mathrm{cm}^{-2} \mathrm{~s}^{-1}$ (for the solar wind exclusively composed of protons). If we assume that all the solar wind energy is deposited in the first centimeter below the nucleus surface, the energy deposited per second in the nucleus of $\mathrm{KB}$ comets by the solar wind is approximately $2.4 \times 10^{7} \mathrm{eV} \mathrm{cm}^{-3} \mathrm{~s}^{-1}$. Over the period of $4.5 \mathrm{~Gy}$ this corresponds to the total dose of $3.4 \times 10^{24} \mathrm{eV} \mathrm{cm}^{-3}$.

For OC comets, we use the solar wind properties from Bagenal et al. (2015) and consider that the solar wind propagates at constant velocity and that the density decreases with the square of the distance to the Sun. If the solar wind energy is deposited in the first centimeter below the surface it corresponds to a deposited energy of $68 \mathrm{eV} \mathrm{cm}^{-3} \mathrm{~s}^{-1}(9.2 \times$ $10^{18} \mathrm{eV} \mathrm{cm}^{-3}$ over $4.5 \mathrm{~Gy}$ ) at the inner edge of the OC (at $20,000 \mathrm{au})$ and of $26 \mathrm{eV} \mathrm{cm}^{-3} \mathrm{~s}^{-1}\left(3.7 \times 10^{17} \mathrm{eV} \mathrm{cm}^{-3}\right.$ over $4.5 \mathrm{~Gy}$ ) at the outer edge of the OC (at 100,000 au). Outside the heliosphere, the low-energy interstellar charged particle will also impact the comet. Such particles may interact with OC comets only as $\mathrm{KB}$ comets are located inside the heliosphere. Measurements by the Voyager probes show shown that the particle count rate drops outside of the heliopause except for energetic cosmic rays (and references therein Krimigis et al. 2019). Considering the uncertainties on the spectrum of these particles, we do not compute the total energy deposited. However, the particle pressure in the heliosheath is dominated by protons with typical energies comprised between 0.03 and $4 \mathrm{MeV}$ (Krimigis et al. 2019 and references therein). Owing to the low flux and low energy of interstellar plasma, it is possible to affirm that the deposition by the observed flux is negligible compared to the deposition by the GCRs.

\subsection{Solar Energetic Particle Events}

We simulated the energy deposited during an SEP event. We considered a super-Carrington SEP event at the location of the $\mathrm{KB}$, which is what would be observed in the early solar system (Airapetian et al. 2016; Fu et al. 2019). As seen in Figure 7 our simulation shows that the energy deposition via SEP events from the young Sun is mainly in the first meter. During such events, the deposition rate is higher than the GCR one in the first $8 \mathrm{~m}$. In the first centimeters below the surface the deposition rate of SEP events is several orders of magnitude higher than the one of GCR. At a depth of $1 \mathrm{~m}$ the deposition rate is approximately two orders of magnitude higher for a super-Carrington SEP event, impacting the comet located in the $\mathrm{KB}$, than for GCRs. For present-day SEP events, the dose rate should be reduced by a factor of $10^{4}$; this is a dose comparable to the GCR one during the event in the first centimeters below the surface and approximately two orders of magnitude lower at a depth of $1 \mathrm{~m}$.

SEP events with a significant amount of particles above $500 \mathrm{MeV}$ are relatively rare now, of the order of two per year. They last for a couple days at most (Kühl et al. 2017) but may 
have been more frequent in the early solar system (Airapetian et al. 2019; Saxena et al. 2019). The total dose they deposit in cometary nuclei is therefore only significant in the first centimeters below the surface. Deeper into the nucleus, the total energy deposited by SEP events over the lifetime of comets is well below the total energy deposited by GCRs.

\section{Conclusion}

Most of the ionizing energy coming from the Sun-EUVXUV (X-ray and ultraviolet), electrons, solar wind fluxes-is deposited in the first meter of a comet (Johnson 1991; Johnson \& Quickenden 1997). Its contribution to the energy deposition in the first centimeters below the surface is significant. In the $\mathrm{KB}$ region the energy deposited by the solar wind in the first centimeter below the surface can be of the same order of magnitude as the energy deposited by GCRs. The irradiation fluxes via solar EUV-XUV, gamma-ray bursts, supernovae, and energetic particles emitted by the Sun at its early stage is much more difficult to constrain as we do not have a precise knowledge of their fluence and/or occurrence frequency. These events deposit most of their energy in the first meter below the cometary surface; the energy deposition at greater depth is dominated by GCRs. The outer surface of the comet below which such particles deposit most of their energy interacts with the ISM during the lifetime of the comet and it is not clear if this interaction will result in the accretion of ISM material or in the erosion of the nucleus surface. In addition, the first meter of a cometary nucleus is quickly removed upon a comet's first close approach to the Sun. We present here the calculations for the dose deposited deeper inside the cometary nucleus, below the first meter. The particles that have a potential to penetrate deeper in the comet are the SEPs and the GCRs. The GCRs deposit energy in nonnegligible amounts in the first $70 \mathrm{~m}$ below the surface. This can change our view of the dynamically young comets, doing their first orbits in the inner solar system and for which the coma is formed from those outer layers that have received a substantial dose of energy. In addition, the knowledge of the dose deposited over time may help understand the evolution of the $\mathrm{KB}$ objects observed by missions such as New Horizons. The effects of the energy deposition result in changes in isotopic composition and cometary chemistry, as described in Paper II.

The work of G.G. and W.B.M. was supported by the Living Breathing Planet project: NASA Astrobiology Institute grant NNX15AE05G. The work of G.G. and V.A. was supported by the NASA Exobiology grant 80NSSC17K0463. Work at BIRA-IASB regarding energetic particle impacts was supported by the Solar-Terrestrial Centre of Excellence, while ROSINA data analysis was supported via PRODEX/ROSINA PEA 90020 and an Additional Researchers Grant (Ministerial Decree of 2014 December 19), thanks to the Belgian Science Policy Office. A.G. thanks FNRS for a FRIA research grant. M. $R$. acknowledges the State of Bern and the Swiss National Science Foundation (SNSF, 200020_182418). H.G. was supported by the Swedish National Space Agency grant 108/ 18 C.J.M. was supported by the Advanced Exploration Systems Division within the NASA Human Exploration and Operations Mission Directorate. G.G. wants to thank L.F.G. for useful discussions. The authors wish to thank an anonymous referee for useful comments.

\section{ORCID iDs}

G. Gronoff (i) https://orcid.org/0000-0002-0331-7076

R. Maggiolo (iD https://orcid.org/0000-0002-5658-1313

V. Airapetian (i) https://orcid.org/0000-0003-4452-0588

J. De Keyser (iD https://orcid.org/0000-0003-4805-5695

M. Rubin (iD https://orcid.org/0000-0001-6549-3318

\section{References}

Agostinelli, S., Allison, J., Amako, K., et al. 2003, NIMPA, 506, 250 Airapetian, V. S., Barnes, R., Cohen, O., et al. 2019, IJAsB, in press Airapetian, V. S., Glocer, A., Gronoff, G., Hébrard, E., \& Danchi, W. 2016, NatGe, 9, 452

Altwegg, K., Balsiger, H., Bar-Nun, A., et al. 2015, Sci, 347, 1261952

Altwegg, K., Balsiger, H., Berthelier, J. J., et al. 2017, RSPTA, 375, 20160253

Badhwar, G. D., \& O’Neill, P. M. 1992, IJRAI, 20, 403

Bagenal, F., Delamere, P. A., Elliott, H. A., et al. 2015, JGRE, 120, 1497

Balsiger, H., Altwegg, K., Bar-Nun, A., et al. 2015, SciA, 1, e1500377

Bardyn, A., Baklouti, D., Cottin, H., et al. 2017, MNRAS, 469, S712

Belyaev, M. A., \& Rafikov, R. R. 2010, ApJ, 723, 1718

Bertaux, J.-L. 2015, A\&A, 583, A38

Bieler, A., Altwegg, K., Balsiger, H., et al. 2015, Natur, 526, 678

Capaccioni, F., Coradini, A., Filacchione, G., et al. 2015, Sci, 347, aaa0628

Carusi, A., Kresak, L., Perozzi, E., \& Valsecchi, G. B. 1985, Long-term Evolution of Short-period Comets (Bristol: Adam Hilger), 272

Cummings, A. C., \& Stone, E. C. 2007, SSRv, 130, 389

Davidsson, B. J. R., Sierks, H., Güttler, C., et al. 2016, A\&A, 592, A63

De Keyser, J., Dhooghe, F., Altwegg, K., et al. 2017, MNRAS, 469, S695

Dhooghe, F., De Keyser, J., Altwegg, K., et al. 2017, MNRAS, 472, 1336

Duncan, M., Levison, H., \& Dones, L. 2004, in Comets II, ed. M. C. Festou,

H. U. Keller, \& H. A. Weaver (Tucson, AZ: Univ. Arizona Press), 193

Feigelson, E. D., \& Montmerle, T. 1999, ARA\&A, 37, 363

Fu, S., Jiang, Y., Airapetian, V., et al. 2019, ApJL, 878, L36

Fulle, M., Altobelli, N., Buratti, B., et al. 2016, MNRAS, 462, S2

Giacalone, J., Drake, J. F., \& Jokipii, J. R. 2012, SSRv, 173, 283

Gronoff, G. 2009, PhD thesis, Université Joseph-Fourier-Grenoble I, https:// tel.archives-ouvertes.fr/tel-00400638

Gronoff, G., Lilensten, J., Desorgher, L., \& Flückiger, E. 2009, A\&A, 506, 955

Gronoff, G., Mertens, C., Lilensten, J., et al. 2011, A\&A, 529, 143

Gronoff, G., Mertens, C. J., Norman, R. B., Straume, T., \& Lusby, T. C. 2016, SpWea, 14, 835

Gronoff, G., Norman, R. B., \& Mertens, C. J. 2015, AdSpR, 55, 1799

Groussin, O., Sierks, H., Barbieri, C., et al. 2015, A\&A, 583, A36

Guilbert-Lepoutre, A., Besse, S., Mousis, O., et al. 2015, SSRv, 197, 271

Iro, N., Gautier, D., Hersant, F., Bockelée-Morvan, D., \& Lunine, J. I. 2003, Icar, 161, 511

Johnson, R. E. 1991, JGR, 96, 17553

Johnson, R. E., \& Quickenden, T. I. 1997, JGR, 102, 10985

Jutzi, M., \& Benz, W. 2017, A\&A, 597, A62

Jutzi, M., Benz, W., Toliou, A., Morbidelli, A., \& Brasser, R. 2017, A\&A, 597, A61

Keller, H. U., Mottola, S., Davidsson, B., et al. 2015, A\&A, 583, A34

Kööp, L., Heck, P. R., Busemann, H., et al. 2018, NatAs, 2, 709

Kovaltsov, G. A., Usoskin, I. G., Cliver, E. W., Dietrich, W. F., \& Tylka, A. J. 2014, SoPh, 289, 4691

Krimigis, S. M., Decker, R. B., Roelof, E. C., et al. 2019, NatAs, 3, 997

Kühl, P., Dresing, N., Heber, B., \& Klassen, A. 2017, SoPh, 292, 10

Läuter, M., Kramer, T., Rubin, M., \& Altwegg, K. 2018, MNRAS, 483, 852 Le Roy, L., Altwegg, K., Balsiger, H., et al. 2015, A\&A, 583, A1

Lis, D. C., Bockelée-Morvan, D., \& Güsten, R. 2019, A\&A, 625, L5

Maggiolo, R., Gronoff, G., Cessateur, G., et al. 2020, ApJ, submitted

Maquet, L. 2015, A\&A, 579, A78

Massironi, M., Simioni, E., Marzari, F., et al. 2015, Natur, 526, 402

Matonti, C., Attree, N., Groussin, O., et al. 2019, NatGe, 12, 157

Matthiä, D., Berger, T., Mrigakshi, A. I., \& Reitz, G. 2013, AdSpR, 51, 329

Mertens, C. J., Meier, M. M., Brown, S., Norman, R. B., \& Xu, X. 2013, SpWea, 11, 603

Morbidelli, A., \& Rickman, H. 2015, A\&A, 583, A43

Mousis, O., Drouard, A., Vernazza, P., et al. 2017, ApJL, 839, L4

Mumma, M. J., Weissman, P. R., \& Stern, S. A. 1993, in Protostars and Planets III, ed. E. H. Levy \& J. I. Lunine (Tucson, AZ: Univ. Arizona Press), 1177 Norman, R. B., Gronoff, G., \& Mertens, C. J. 2014, JGRA, 119, 452

O'Neill, P. M., Golge, S., \& Slaba, T. C. 2014, Badhwar-O'Neill 2014 Galactic Cosmic Ray Flux Model Description, NASA Technical Report 
Pätzold, M., Andert, T., Hahn, M., et al. 2016, Natur, 530, 63

Pätzold, M., Andert, T. P., Hahn, M., et al. 2019, MNRAS, 483, 2337

Pavlov, A. A., Pavlov, A. K., Ostryakov, V. M., et al. 2014, JGRE, 119, 1390

Poluianov, S., Kovaltsov, G. A., \& Usoskin, I. G. 2018, A\&A, 618, A96

Portegies Zwart, S., Pelupessy, I., van Elteren, A., Wijnen, T. P. G., \& Lugaro, M. 2018, A\&A, 616, A85

Quirico, E., Moroz, L. V., Schmitt, B., et al. 2016, Icar, 272, 32

Rickman, H., Marchi, S., A’Hearn, M. F., et al. 2015, A\&A, 583, A44

Rodríguez-Gasén, R., Aran, A., Sanahuja, B., Jacobs, C., \& Poedts, S. 2014, SoPh, 289, 1745

Rotundi, A., Sierks, H., Della Corte, V., et al. 2015, Sci, 347, aaa3905

Rubin, M., Altwegg, K., Balsiger, H., et al. 2015, Sci, 348, 232

Saxena, P., Killen, R. M., Airapetian, V., et al. 2019, ApJL, 876, L16

Schwartz, S. R., Michel, P., Jutzi, M., et al. 2018, NatAs, 2, 379
Sheel, V., Haider, S. A., Withers, P., et al. 2012, JGRA, 117, A05312

Simnett, G. M. 2017, Energetic Particles in the Heliosphere (Berlin: Springer), 189

Smart, D. F., Shea, M. A., \& McCracken, K. G. 2006, AdSpR, 38, 215

Stern, S. A. 1988, Icar, 73, 499

Stern, S. A. 2003, Natur, 424, 639

Stern, S. A., \& Shull, J. M. 1988, Natur, 332, 407

Stern, S. A., \& Weissman, P. R. 2001, Natur, 409, 589

Torres, D. F., Cillis, A., Lacki, B., \& Rephaeli, Y. 2012, MNRAS, 423, 822

Velinov, P. I. Y., \& Mateev, L. 2008, AdSpR, 42, 1586

Wolff, E. W., Bigler, M., Curran, M. A. J., et al. 2012, GeoRL, 39, L08503

Wurz, P. 2005, in Proc. 11th European Solar Physics Meeting, The Dynamic Sun: Challenges for Theory and Observations, ed. D. Danesy, S. Poedts, A. De Groof, \& J. Andries (Paris: ESA), 441 